\title{
A CORRECTION TO: DISCRETE OPEN AND CLOSED MAPPINGS ON GENERALIZED CONTINUA AND NEWMAN'S PROPERTY
}

\section{F. MCAULEY AND E. E. ROBINSON}

In this paper, domains have compact closures.

On pp. 1087 and 1093, lines 6- and 11-, there is no homeomorphism $h$ defined on $M(X)$ onto $M(X)$ having the properties listed.

The proof of Lemma 5.2 has an error on p. 1107 which can be easily corrected.

On p. 1108 , line $9, \sigma$ commutes on $n$-chains with the special projections.

On p. 1109, line 13-, delete "special”.

Theorem 6.2 should read as follows: Suppose that $X$ is an $n$-dimensional generalized continuum. Furthermore, for each domain $A$ in $X, \bar{A}$ compact, the Cech homology group,

$$
\stackrel{\vee}{H}_{n}\left(X, X-A, Z_{p}\right) \cong Z_{p} \text { for the prime } p>1 \text {. }
$$

Then $X$ has Newman's Property with respect to $C(p)$.

In Theorem 6.2, we consider the class $C(p), p>1$, of all finite-to-one open and closed mappings $f$ on an $n$-dimensional generalized continuum such that

(1) $f$ maps $X$ onto a generalized continuum $Y_{f}$,

(2) if $F=f\left(B_{f}\right)$, then $\operatorname{dim} F<n$,

(3) $N(f)=p$, and

(4) $\{x \mid N(x, f)=N(f)\}$ is dense in $X$.

We could consider $C(k)$ where $p$ is the smallest prime divisor of $k$.

The projection $\pi: N(B) \rightarrow N(U)$ takes an essential $n$-cycle $Z^{n}(B) \bmod X$ - $D$ to an essential $n$-cycle $Z^{n}(U) \bmod X-D$.

The Lebesque number $\epsilon$ of $B$ is relative to the subcollection $B$ each of whose members meets $\bar{A}$.

Note that $\sigma \sigma Z^{n}\left(G_{f}\right)=x^{2} Z^{n}\left(G_{f}\right)$. Either $x=0$ or $x=1$ in case $p=2$. It follows that $x=0$. For $p>2$, a similar argument yields that $x=0$. Thus, $\sigma Z^{n}\left(G_{f}\right)=0$. This means that the sum of the coefficients of the $n$-simplices in $Z^{n}\left(G_{f}\right)$ which belong to the same distinguished family is 0 $\bmod p$. Indeed, each $n$-simplex in $Z^{n}\left(G_{f}\right)$ belongs to a distinguished family

Received February 14, 1985. 
consisting of $p n$-simplices no one of which meets $f^{-1}(F)$. Thus, $\pi Z^{n}\left(G_{f}\right)$ $=0$ (the 0 -cycle) on $A$. By construction of $G_{f}, \pi Z^{n}\left(G_{f}\right) \neq 0$ on $A$. Hence, it is false that

$$
\operatorname{diam} f^{-1} f(x)<\epsilon \text { for each } x \in A .
$$

The theorem is proved.

In Corollary 6.21, the conclusion is that $X$ has Newman's Property with respect to $C(p)$ as defined above.

In Corollary 6.22, the conclusion is that $X$ has Newman's Property (as stated in Section 3), i.e., with respect to the class of all finite-to-one open and closed mappings $f$ on $X$ with $N(f)>1$.

The statement that $\sigma$ takes essential $n$-cycles to essential $n$-cycles is false and is never used in the paper. 